\title{
Strategies of pain reduction during the bone marrow biopsy
}

\author{
Nikolaj Hjortholm • Emil Jaddini • \\ Kazimierz Hałaburda • Emilian Snarski
}

Received: 10 October 2012 / Accepted: 21 November 2012/Published online: 6 December 2012

(C) The Author(s) 2012. This article is published with open access at Springerlink.com

\begin{abstract}
Examination of the bone marrow biopsy and aspirate allows diagnosis and assessment of various conditions such as primary hematologic and metastatic neoplasms, as well as nonmalignant disorders. Despite being performed for many years, according to many different protocols, the procedure still remains painful for the majority of patients. This paper summarizes the current knowledge of pain reduction measures in the bone marrow biopsy and aspiration.
\end{abstract}

Keywords Pain $\cdot$ Bone marrow $\cdot$ Biopsy $\cdot$ Aspiration · Hematology

\section{Introduction}

The bone marrow biopsy and aspiration (BMBA) is an essential diagnostic approach to the diagnosis of hematological disorders [43]. It was developed in the beginning of the nineteenth century, allowing doctors to diagnose marrow disorders in patients $[16,33]$. The procedure evolved in the following years with focus on easier and repeatable collection of bone marrow, leading to full standardization of the procedure in the 1970s [7, 14, 22]. Local anesthesia was used to reduce the pain in patients undergoing biopsy. Despite the progress in medicine, there was not much focus on studies considering the pain during the bone marrow biopsy. The information on prevalence, predicting factors, and prevention of pain associated with the procedure is very limited, and no effective guidance for the technique itself was so far developed [43, 45]. The standard methods of performing BMBAs have remained almost unchanged for

N. Hjortholm $(\bowtie) \cdot$ E. Jaddini $\cdot$ K. Hałaburda $\cdot$ E. Snarski Department of Hematology, Oncology and Internal Medicine, Medical University of Warsaw, Warsaw, Poland

e-mail: hjortholmnikolaj@gmail.com decades. Nevertheless, newer studies acknowledge the lack of information on pain reduction during a BMBA procedure [45]. As the importance for more information in this field is being recognized, there are no clear instructions in how to use data on pain-causing factors to prevent or deal with pain in patients undergoing this type of biopsy to date.

Still, the limited trials undertaken to lessen the pain connected with the procedure did not change clinical practice, and pain remains a heavy burden to many hematologic patients [28]. As medicine becomes more patient-oriented and emphasis is being placed on patient well-being, we strive to decrease the pain associated with medical procedures. So far, there are no set guidelines to ameliorate pain resulting from a BMBA. In this review, we analyze current practice to reduce pain during the procedure and summarize the simplest ways for pain reduction during a bone marrow biopsy.

\section{Bone marrow biopsy and aspiration}

Usually, a bone marrow biopsy and aspiration is performed in a hospital or an outpatient clinic by a trained physician. Typical biopsy sites are the posterior and anterior superior iliac crest, but the sternum can also be used for aspiration. Before the biopsy, a local anesthetic is injected into the subcutaneous tissue to numb the area and reduce pain. The patient may also receive systemic analgesia and/or antianxiety medications [35]. A needle is then introduced through the skin into the bone, and a sharp pain may be felt as the needle is inserted into the bone. The bone marrow biopsy is usually, but not always, performed first. By applying manual pressure, the needle is propelled through the bony cortex. If the biopsy is performed in the posterior or anterior iliac crest, the larger trephine biopsy (TB) needle is used to collect a cylindrical sample of solid bone marrow. The TB 
needle is removed, and, with a syringe attached to a needle, a small amount of marrow fluid is removed from the bone marrow. Finally, the needle is removed, and pressure is applied to the biopsy site to stop possible bleeding.

The posterior superior iliac crest is a preferred site for the BMBA due to safety, comfort, and convenience. The anterior superior iliac crest is less preferred due to increased occurrence of pain and difficulty in sampling [21]. The approach to the sternum aspiration varies between centerssome centers use the sternum as the primary site for aspiration when there is no need for additional TB, while other centers use it only as a last resort in patients who, for medical reasons, cannot be biopsied otherwise. The sternal aspiration benefits are weighted against the risks associated with accidentally entering of the underlying mediastinum or causing a fatal episode of cardiac tamponade [32, 34]. Cases of hemorrhage have been reported, however, with very rare frequency $[5,42]$. The other reason for not using the sternum as the primary site for bone marrow aspiration is the observation that sternal aspirations were reported by the patients to be the most painful localization for aspiration [25]. However, there are little available data on that topic. In infants younger than 1 year, the tibia is sampled, and the procedure is done under general anesthesia $[4,40]$. The BMBA from the posterior iliac crest typically takes approximately $10-30 \mathrm{~min}$, and the patient is usually released home after an additional hour of observation to exclude any immediate local complications. If an intravenous sedation has been administered, extra time is needed for preparation and post-procedure care. Discomfort, mild tenderness, and minor bleeding may occur at the site of the biopsy. Complications, such as infections, serious bleeding, or breaking of the needle, are extremely rare, and bone marrow examination is considered a safe procedure with low risk of morbidity [36, 42].

\section{Pain-influencing factors}

There are many independent factors that were studied in possible connection to pain in BMBAs. These include gender, age, body mass index (BMI), level of education, information prior to procedure, previous BMBA experience, indication for BMBAs, site of BMBAs, the experience of the operator, duration, and difficulty of the procedure [44]. The results of the studies are often contradictory when separate characteristics, such as BMI, age, or operator experience, are taken into account.

The correlation between duration and difficulty of the procedure in relation to pain has been recorded in several studies [26, 44]. Intensity of pain was significantly reduced when the procedure was performed by an experienced physician or took less than $10 \mathrm{~min}$, since the duration of the procedure is equivalent to a higher visual analog scale score
[44]. The experience of the physician was also shown to reduce the time of a BMBA [26]. However, in other studies, the experience of a physician shows no effect on pain [11, 23]. Patients attempting one biopsy, compared to repetitive biopsies, also reported fewer episodes of unbearable pain [11], especially a long duration of puncture was a significant independent predictor of pain intensity [26]. An experienced physician can usually perform a bone marrow biopsy with minimal pain and discomfort. This in part can be explained by a reduction of time needed for the procedure, as patients undergoing lengthy procedures report higher pain scores than patients undergoing shorter uncomplicated procedures. Also, experienced physicians are typically better at overcoming the technical problems and decrease the need of multiple biopsies in a given patient.

Another factor influencing pain during the biopsy is the BMI of a patient. Some suggest that BMI and age play only a minor role to no direct association with episodes of severe pain $[11,26]$, while others claim that these are key factors in the pain experience [44]. This can be, in part, explained that the posterior superior iliac crest can be inaccessible for morbid obese patients (BMI $>30 \mathrm{~kg} / \mathrm{m}^{2}$ ) that tend to undergo the biopsy of the sternum, which has an increased risk of adverse effects and severe pain perception $[4,5]$.

Pain during a prior BMBA and anticipatory anxiety had a significant impact on pain during a subsequent examination. Patients experiencing severe pain in the first biopsy would be more prone to report unbearable pain in the following BMBAs [11,23]. Since a negative experience can lead to fear and emotional distress, any future BMBA may become an increasing problem for patients. This is important because, in several cases, procedures are frequently repeated. Therefore, it is important not to traumatize the patient for the future. Patients experiencing high anxiety before the BMBA tend to score higher levels of pain [8]. Inadequate information prior to the procedure also seems to be a key factor in some studies [11]. Many patients claim that they do not get adequate information on adverse effects before the puncture [3].

Bone marrow biopsies often remain a heavy burden for patients. The research so far has concentrated on painassociated factors rather than pain-reducing measures. However, most of the pain predictors show us the areas of concern and for future interventions. The experience of the physician seems to be the key influencing factor in most of the studies.

\section{Methods of pain reduction}

Pharmacologic

When a BMBA is performed in adults, a local anesthetic (LA), usually lidocaine or a similar drug, is used to numb 
the area of the skin and periosteum at the puncture site before the procedure. Lidocaine can be given along with sodium bicarbonate to reduce the burning pain of the lidocaine solution [35]. In case of an adverse reaction to the medication, an alternative drug, like chloroprocaine or bupivacaine hydrochloride can be used [35]. Articaine, a LA that is able to penetrate the bone, can also be used, however, studies have shown that the drug is not superior to lidocaine, and that patients do not report lower pain scores [27]. Other drugs may be used for local anesthesia such as mepivacain; however, there are very limited data to support the use of one drug over another in bone marrow biopsies.

Intravenous sedation (IVS) with drugs such as lorazepam, midazolam, or diazepam is often administered in addition to LA [10, 15]. Besides reducing anxiety and pain perception, IVS has shown to produce a retrograde amnesia for the procedure in many patients [30]. These drugs are commonly requested by patients that experienced severe pain during their first bone marrow biopsy (which underlines the importance that the first biopsy is as painless as possible). In a different study, a combination of hydromorphone and lorazepam administered 90 min prior to a BMBA showed a lower pain perception and retrograde amnesia in half of the patients [13]. According to several studies, patients receiving IVS tend to be more often willing to undergo a future BMBA, compared to patients receiving only LA [31]. Apart from the favorable effects, IVS administration has serious drawbacks, like prolongation of the hospital stay, requirement of additional staff and equipment, and furthermore, it contributes to an increase in incidence of adverse effects. Before administration, the beneficial effects of IVS need to be balanced against the adverse effects for each given patient. When given the choice between LA only and LA/IVS, patients choosing LA were more satisfied with their choice. However, patients that received IVS alongside LA had a lower pain score compared to patients receiving LA only [17]. We have to remember that many times the patients experiencing severe pain during the first bone marrow biopsy will ask for IVS. Personal experience shows that when biopsy is performed in such patients by an experienced physician that uses only LA, the IVS will not be requested in further biopsies if the patients who felt pain during biopsy are satisfied afterwards.

Since LA does not prevent the transient pain experienced during suction, premedication with a centrally acting opioid analgesic, like tramadol, can be used to reduce the pain intensity significantly, indicating that the pain provoked by vacuum aspiration of bone marrow is opioid-responsive [44]. In comparison to other opioids, tramadol lacks the unwanted effects on the cardiovascular and respiratory system and is therefore better tolerated $[12,37]$. With a $50-\mathrm{mg}$ dose used prior to the biopsy, the pain could be reduced, and there were no major side effects reported in the study patients. Another study indicates the use of deep sedation in the form of midazolam, fentanyl, and propofol for pain reduction [9].

The most recent studies have shown some reduction in pain intensity with the usage of nitrous oxide/oxygen $\left(\mathrm{N}_{2} \mathrm{O} / \mathrm{O}_{2}\right)$ or nitrous oxide alone [24]. This gas may be used for pain relief in BMBAs [20, 41]. Patients self-administer the gas a couple of minutes before and during the procedure when discomfort is experienced [23]. The benefits of this approach are the low cost, no hospitalization, and less nursing or medical supervision. In a study, it was shown that $93 \%$ of pediatric patients undergoing various procedures with a nitrous oxide/oxygen mixture, would choose this again, if the procedure had to be repeated in the future [2]. However, adverse effects like diffusion hypoxia have been reported [26]. In a different study [43], patients were offered a choice between LA alone or LA in combination with $\mathrm{N}_{2} \mathrm{O} / \mathrm{O}_{2}$. A pain reduction was noted in the $\mathrm{LA} / \mathrm{N}_{2} \mathrm{O} / \mathrm{O}_{2}$ group, but the results were not statistically significant. However, $84 \%$ of the patients using this combination would request it again for a future procedure [43].

In conclusion, premedication with a LA alone or in combination with IVS did reduce pain but did not prevent unbearable pain [11]. Tramadol can be used to further reduce the intensity of pain, but severe pain may still occur in some patients [46].

\section{Nonpharmacologic}

One of the important pain-influencing factors during BMBAs is the patients' anxiety before the procedure. Establishing trust by being honest, providing accurate information in terms the patient can understand, and ensuring comfort and privacy can lower the patients' anxiety prior to the procedure. A survey of data clearly indicates the influence of information provided by the physician on pain during a procedure [11]. Often underestimated, good information prior to procedure could be an effective and simple way to reduce pain during examinations, since inadequate information at any time shows a trend towards an association with unbearable pain. Patients who reported to have received information about the procedure or who had previous personal experience with bone marrow examination could arrange a mental strategy and experience unbearable pain less often [11].

Another approach is the use of different types of biopsy devices. Development of new equipment, such as biopsy needles with internal snares to capture bone marrow, reduces the discomfort experienced by the patient $[18,19]$ and allows easier biopsy for the physician if used properly. In addition, a new mechanical device, the OnControl Bone Marrow Biopsy System, was introduced recently. The mechanical device was assessed in a small randomized trial, in which it was shown to cause similar pain compared to the 
conventional biopsy needles, however with possibility of more complications, even though the biopsy done with the powered device took half the time compared to the manual biopsy [6]. The positive effect of automated biopsy needle was a reduction of the residual pain the day after the biopsy. Unfortunately, the data coming from a limited number of patients cannot be well interpreted, as it is impossible to randomize the operator's skills to perform the bone marrow biopsy with a normal device versus a new device that is provided by the sponsor of the trial.

Also, alternative ways of pain reduction were explored in different centers to reduce the discomfort associated with a BMBA. For example, hypnosis has been used as an adjunct to local anesthetics and showed to be beneficial $[1,29]$. In one study, hypnosis showed to lower the anxiety during the procedure but did not show any significant decrease in pain [39]. Cognitive behavioral therapy has also been compared to hypnosis but has not been as effective [29]. Other variants are used, such as music therapy or art therapy (like nature screens combined with nature sounds), to provide a distraction for the patient and to decrease the level of anxiety [38].

\section{Strategy for pain reduction during bone marrow biopsy}

So far, there is no formal recommendation on how to effectively reduce pain during the bone marrow biopsy. However, the data collected during different trials and the experience from different centers show that simple conclusions can be drawn. Firstly, the emphasis should be focused on patient information. The doctors should inform the patient about the procedure and provide necessary information on what will be done. Secondly, the first biopsy should preferably be performed by an experienced operator as this is the point where the greatest anxiety is felt, and pain during that first procedure was shown to be connected with pain in the following biopsies. When possible, we would recommend sending psychologically fragile patients undergoing their first BMBA to a physician in a center known to perform the least painful BMBA. Additionally, better pharmacological ways for patient anesthesia and sedation should be explored. The patients should be carefully anesthetized, and the local anesthetic, allowed time for proper infiltration of the puncture site. Insufficient local anesthesia is the most easily avoidable mistake during bone marrow biopsies. Short sedations can be offered to patients preferring pain relief, if the ward has the capacity of providing the possibility for further observation. For patients experiencing severe pain during the marrow suction, tramadol can be given before the procedure, as it was shown to significantly reduce this pain. The patient should be asked when the strongest pain was experienced during the previous procedure, as this provides the operator with information on possible paincausing factors and allows in many cases the improvement in following biopsies. There is a little chance that formal recommendations for BMBAs will be made at any time soon, as there are very little data arising from trials in this field of medicine. We can just hope that slow progress in this field and implementation of the data already present will lead to less painful biopsies in the future.

\section{Conclusions}

Is the bone marrow biopsy pain something that can be reduced? Yes. However, the issue of pain associated with biopsy is under-researched. There are only a handful of studies that assess the different factors associated with pain, and even less randomized clinical trials have been performed in this area. What we know is that, on average, the pain associated with a BMBA is short-lived, mild to moderate, and less than anticipated. However, for some patients, a BMBA is a severely painful process, and, as the unbearable pain score is closely related to the difficulty of the BMBA and the experience of the performing physician, this is an issue that could be targeted in future clinical trials. However, as trials are expensive and hard to run, we propose a simple online tool that helps physicians learn how to reduce the pain in patients undergoing bone marrow biopsy. The project can be accessed on the webpage www.painlessbiopsy.wordpress. com. The idea is to provide physicians with instant feedback on the level of pain felt during the biopsy and possibility to access the knowledge of the experienced colleagues on the topic of bone marrow biopsy. The use of analgesics and sedatives leads to the decrease in discomfort and pain during BMBAs but carries with them added risk of complications. One point which is relatively easy to pursue in most of clinical situations is providing the patient with good and adequate information. Although there are only limited approaches, both pharmacologic and nonpharmacologic, available to manage the pain perception during a BMBA; our observation suggests that they can be easily adopted into clinical practice and could be a great help for patients undergoing bone marrow biopsy.

Conflict of interest The authors declare that they have no conflict of interest.

Open Access This article is distributed under the terms of the Creative Commons Attribution License which permits any use, distribution, and reproduction in any medium, provided the original author(s) and the source are credited.

\section{References}

1. Accardi MC, Milling LS (2009) The effectiveness of hypnosis for reducing procedure-related pain in children and adolescents: a comprehensive methodological review. J Behav Med 32(4):328-339 
2. Annequin D, Carbajal R, Chauvin P, Gall O, Tourniaire B, Murat I (2000) Fixed $50 \%$ nitrous oxide oxygen mixture for painful procedures: a French survey. Pediatrics 105(4):E47

3. Audran M, Maury E, Bouvard B, Legrand E, Baslé MF, Chappard D (2012) Is transiliac bone biopsy a painful procedure? Clin Nephrol 77(2):97-104

4. Bain BJ (2001) Bone marrow trephine biopsy. J Clin Pathol 54 (10): $737-742$

5. Bain BJ (2003) Bone marrow biopsy morbidity and mortality. Br J Haematol 121:949-951

6. Berenson et al (2011) Using a powered bone marrow biopsy system results in shorter procedures, causes less residual pain to adult patients, and yields larger specimens. Diag Pathol 6(23)

7. Birch CD, Fischer S, Zibell A, Jensen ME (1982) Diagnostic bone marrow studies extended routinely by iliac crest biopsy, using the method of Schaadt-Fischer. Acta Pathol Microbiol Immunol Scand 90:229-234

8. Brunetti GA, Tendas A, Meloni E, Mancini D et al (2011) Pain and anxiety associated with bone marrow aspiration and biopsy: a prospective study on 152 Italian patients with hematological malignancies. Ann Hematol 90(10):1233-1235

9. Burkle CM, Harrison BA, Koenig LF, Decker PA, Warner DO, Gastineau DA (2004) Morbidity and mortality of deep sedation in outpatient bone marrow biopsy. Am J Hematol 77(3):250-256

10. Chakupurakal G, Delgado J, Nikolousis E, Pitchapillai S, Allotey D, Holder K, Bratby L, de la Rue J, Milligan DW (2008) Midazolam in conjunction with local anaesthesia is superior to Entonox in providing pain relief during bone marrow aspirate and trephine biopsy. J Clin Pathol 61:1051-1054

11. Degen C, Christen S, Rovo A, Gratwohl A (2010) Bone marrow examination: a prospective survey on factors associated with pain. Ann Hematol 89:61924

12. Desmeules JA (2000) The tramadol option. Eur J Pain 4:15-21

13. Dunlop TJ, Deen C, Lind S, Voyle RJ, Prichard JG (1999) Use of combined oral narcotic and benzodiazepine for control of pain associated with bone marrow examination. South Med J 92(5):477-480

14. Ellis LD, Jensen WN, Westerman MF (1964) Needle biopsy of bone and bone marrow. An experience with 1,445 biopsies. Arch Intern Med 114(2):213-221

15. Friedman AG, Mulhern RK, Fairclough D et al (1991) Midazolam premedication for pediatric bone marrow aspiration and lumbar puncture. Med Pediatr Oncol 19(6):499-504

16. Ghedini G (1908) Studi sulla patologia del midollo osseo umano vivente. I. Puntura explorative tecnica. Clin Med Ital 47:724

17. Giannoutsos I, Grech H, Maboreke T et al (2004) Performing bone marrow biopsies with or without sedation: a comparison. Clin Lab Haematol 26:201-204

18. Goldenberg AS, Rishton M (1999) Bone-marrow biopsy needle incorporating a snare-coil specimen-capturing device: description and preclinical studies. Biomed Instrum Technol 33(6):522-529

19. Goldenberg AS, Tiesinga JJ (2001) Clinical experience with a new specimen capturing bone marrow biopsy needle. Am J Hematol 68 (3):189-193

20. Gudgin EJ, Besser MW, Craig JIO (2008) Entonox as a sedative for bone marrow aspiration and biopsy. Int J Lab Hema 30(1):65-67

21. Hernandez-Garcia MT, Hernandez-Nieto L, Perez-Gonzalez E, Brito-Barroso ML (1993) Bone marrow trephine biopsy: anterior superior iliac spine versus posterior superior iliac spine. Clin Lab Haematol 15(1):15-19

22. Jamshidi K, Windschitl HE, Swaim WR (1971) A new biopsy needle for bone marrow. Scand J Haematol 8(1):69-71

23. Johnson H, Burke D, Plews C, Newell R, Parapia L (2008) Improving the patient experience of a bone marrow biopsy - an RCT. J Clin Nurs 17(6):717-725

24. Kanagasundaram SA, Lane LJ, Cavalletto BP, Keneally JP, Cooper MG (2001) Efficacy and safety of nitrous oxide in alleviating pain and anxiety during painful procedures. Arch Dis Child 84(6):492495

25. Knowles S, Hoffbrand AV (1980) Bone-marrow aspiration and trephine biopsy. Br Med J 281(6234):204-205

26. Kuball J, Schüz J, Gamm H, Weber M (2004) Bone marrow punctures and pain. Acute Pain 6(1):9-14

27. Kuivalainen AM, Niemi-Murola L, Widenius T et al (2010) Comparison of articaine and lidocaine for infiltration anaesthesia in patients undergoing bone marrow aspiration and biopsy. Eur $\mathrm{J}$ Pain 14(2):160-163

28. Liden Y, Landgren O, Arner S, Sjolund K, Johansson E (2009) Procedure related pain among adult patients with hematologic malignancies. Acta Anaesthesiol Scand 53:354-363

29. Liossi C, Hatira P (1999) Clinical hypnosis versus cognitive behavioral training for pain management with pediatric cancer patients undergoing bone marrow aspirations. Int J Clin Exp Hypn 47(2):104-116

30. Milligan DW, Howard MR, Judd A (1987) Premedication with lorazepam before bone marrow biopsy. J Clin Pathol 40(6):696-698

31. Park SH, Bang SM, Nam E, Cho EK, Shin DB, Lee JH, Ahn JY (2008) A randomized double-blind placebo-controlled study of low-dose intravenous Lorazepam to reduce procedural pain during bone marrow aspiration and biopsy. Pain Med 9(2):249-252

32. Pascali VL, Lazzaro P, Fiori A (1987) Is sternal bone marrow needle biopsy still a hazardous technique? Report of three further fatal cases. Am J Forensic Med Pathol 8(1):42-44

33. Peabody FW (1927) Pathology of bone marrow in pernicious anemia. Am J Pathol 3(3):179-202.9

34. Puschel K, Mattern R, Mittmeyer HJ, Schneider V (1985) Errors and hazards: fatalities through sternal puncture. Dtsch Med Wochenschr 110(42):1611-1613

35. Riley RS, Hogan TF, Pavot DR et al (2004) A pathologist's perspective on bone marrow aspiration and biopsy: I. Performing a bone marrow examination. J Clin Lab Anal 18(2):70-90

36. Rywlin AM (1976) Histopathology of the bone marrow. Little, Brown \& Co, Boston, pp 126-132

37. Scott LJ, Perry CM (2000) Tramadol: a review of its use in perioperative pain. Drugs 60(1):139-176

38. Shabanloei R, Golchin M, Esfahani A, Dolatkhah R, Rasoulian M (2010) Effects of music therapy on pain and anxiety in patients undergoing bone marrow biopsy and aspiration. AORN J 91(6):746-751

39. Snow A, Dorfman D, Warbet R, Cammarata M, Eisenman S, Zilberfein F, Isola L, Navada S (2012) A randomized trial of hypnosis for relief of pain and anxiety in adult cancer patients undergoing bone marrow procedures. J Psychosoc Oncol 30(3):281-293

40. Sola MC, Rimsza LM, Christensen RD (1999) A bone marrow biopsy technique suitable for use in neonates. Br J Haematol 107(2):458-460

41. Steedman B, Watson J, Ali S, Shields ML, Patmore RD, Allsup DJ (2006) Inhaled nitrous oxide (Entonox) as a short acting sedative during bone marrow examination. Clin Lab Haematol 28(5):321-324

42. Steinke B (1992) Complications after bone marrow biopsy. Dtsch Med Wochenschr 19(117(25)):1003-1004

43. Talamo G, Liao J, Bayerl MG, Claxton DF, Zangari M (2010) Oral administration of analgesia and anxiolysis for pain associated with bone marrow biopsy. Support Care Cancer 18(3):301-305

44. Vanhelleputte P, Nijs K, Delforge M et al (2003) Pain during bone marrow aspiration: prevalence and prevention. J Pain Symptom Manage 26(3):860-866

45. Watmough S, Flynn M (2011) A review of pain management interventions in bone marrow biopsy. J Clin Nurs 20(5-6):615-623

46. Wolanskyj AP, Schroeder G, Wilson PR (2000) A randomized placebo-controlled study of outpatient premedication for bone marrow biopsy in adults with lymphoma. Clin Lymphoma 1 (2):154-157 